\title{
Effects of Intravenous Landiolol on Heart Rate and Outcomes in Patients with Atrial Tachyarrhythmias and Acute Decompensated Heart Failure: A Single-Center Experience
}

\author{
Yuko Matsui $^{1}$ - Atsushi Suzuki ${ }^{1} \cdot$ Tsuyoshi Shiga $^{1} \mathbb{D} \cdot$ Kotaro Arai $^{1} \cdot$ Nobuhisa Hagiwara $^{1}$
}

Published online: 27 February 2019

(c) The Author(s) 2019

\begin{abstract}
Objective Atrial tachyarrhythmias (ATAs) lead to clinical deterioration and worsening heart failure (HF) in patients with acute decompensated HF (ADHF). We evaluated the effects of the ultrashort-acting intravenous $\beta 1$-blocker landiolol on the heart rate, hemodynamics, and outcome in patients with ATAs and ADHF.

Methods A total of 67 consecutive hospitalized patients with ATAs and ADHF who were treated with landiolol (36 males, $67 \pm 12$ years) were included in this single-center retrospective study. The primary endpoints were changes in heart rate and systolic blood pressure from baseline during intravenous landiolol administration. The secondary endpoints included restoration of sinus rhythm and outcomes.

Results The median maintenance dose of intravenous landiolol was 3.0 (range 1.0-12.0) $\mu \mathrm{g} / \mathrm{kg} / \mathrm{min}$ and the median treatment duration was 5 (range 1-24) days. Intravenous landiolol reduced heart rate $(141 \pm 17$ beats/min at baseline to $99 \pm 20$ beats/ min at $6 \mathrm{~h}, P<0.001$ ) without a marked reduction in blood pressure or deterioration of HF. During landiolol treatment, 15 (22\%) patients had spontaneously restored sinus rhythm. Eight patients experienced in-hospital death, and 41 (69\%) were discharged with sinus rhythm. During the follow-up of $16 \pm 12$ months, patients with sinus rhythm showed a lower frequency of rehospitalization due to worsening HF than patients with ATAs (5/41 vs. 7/18, $P=0.019)$.

Conclusions Our results showed that intravenous landiolol reduces the heart rate without markedly decreasing blood pressure in patients with ATAs and ADHF. Approximately $70 \%$ of the discharged patients were in sinus rhythm, and these patients showed a lower frequency of rehospitalization due to worsening HF (UMIN-CTR no. UMIN000033650).
\end{abstract}

\section{Key Points}

Atrial tachyarrhythmias lead to clinical deterioration and increase the risk of mortality and morbidity in patients with heart failure.

Intravenous landiolol, an ultrashort-acting intravenous $\beta 1$-blocker, reduced the heart rate without markedly reducing blood pressure in patients with atrial tachyarrhythmias and acute decompensated heart failure.

Approximately $70 \%$ of the discharged patients were in sinus rhythm; these patients showed a lower frequency of death and rehospitalization due to worsening heart failure than patients with atrial tachyarrhythmias.

Tsuyoshi Shiga

shiga.tsuyoshi@twmu.ac.jp

1 Department of Cardiology, Tokyo Women's Medical University, 8-1 Kawada-cho, Shinjuku-ku, Tokyo 162-8666, Japan

\section{Introduction}

Atrial tachyarrhythmias (ATAs), including atrial fibrillation, atrial flutter and fast atrial tachycardia, frequently occur in patients with heart failure (HF) [1-4]. It is recognized that ATAs lead to clinical deterioration and worsen HF [5-7]. ATAs also increase the risk of mortality and morbidity in patients with HF, particularly in those with advanced HF [8-11]. The development of ATAs causes an uncontrolled heart rate with a shortened filling time and provocation of tachycardia-induced cardiomyopathy; an absent atrial kick and irregular ventricular rhythm lead to decreased cardiac output and consequently worsened HF [5-7].

Therefore, the conversion and prevention of ATAs are important in the management of HF. Electrical cardioversion for persistent ATAs during critical illness is effective for restoring sinus rhythm; however, it often requires multiple attempts and is associated with a high recurrence among patients in whom initial cardioversion is successful [12]. If electrical cardioversion fails to restore sinus rhythm, the 
management of these patients remains challenging. Current European Society of Cardiology (ESC) guidelines recommend that intravenous digoxin or amiodarone should be administered to address a slow ventricular rate in patients with rapid ATAs and HF during hemodynamic instability [13]. However, the actions of these drugs are often delayed due to their pharmacokinetic properties.

Landiolol is an ultrashort-acting intravenous $\beta 1$-blocker that was developed and has been used in Japan for the treatment of ATAs and is currently available in Europe [14-16]. Landiolol shows a very high cardioselectivity [17] that is 8- to 12-fold greater than that of esmolol [15, 16]. In addition, landiolol exhibits a less negative inotropic effect and a lower reduction of blood pressure than esmolol in rabbits [18]. The pharmacokinetic properties of landiolol indicate that it is rapidly metabolized in plasma by pseudocholinesterase and has a very short elimination half-life of approximately 4 min $[15,16]$. Recently, landiolol has been reported to be useful for heart rate control in AF patients with HF or reduced left ventricular (LV) systolic dysfunction [19]. However, the effect of landiolol on ATAs complicated with acute decompensated HF (ADHF) has not yet been established. The aim of this study was to evaluate the effects of intravenous landiolol on the heart rate, hemodynamics, and outcome in patients with ATAs and ADHF.

\section{Methods}

\subsection{Patients}

A total of 67 consecutive patients with ATAs who were admitted to Tokyo Women's Medical University Hospital due to ADHF and treated with landiolol from May 2014 to December 2017 were retrospectively included in this analysis. This study was approved by the Institutional Rview Board of Tokyo Women's Medical University.

\subsection{Data Collection}

To identify patients who had been administered intravenous landiolol, we first searched automated inpatient accounting databases. We subsequently confirmed that the patients had a diagnosis of ATAs and ADHF by examining the electronic patient medical records.

ADHF included de novo HF (in the absence of a prior history of HF) and worsening HF (if a previous decompensation or hospitalization for HF was documented). ADHF was defined as the appearance of new or progressive symptoms and signs of decompensated HF, such as significant weight gain, dyspnea, fatigue, pulmonary rales, hepatic congestion, and lower extremity edema, and the unplanned addition of oral or intravenous loop diuretic drugs, an increased dose of oral loop diuretics, the addition of a thiazide diuretic drug to loop diuretics, or the need for treatment with intravenous vasodilators, intravenous inotropes, or intra-aortic balloon pumping.

Data on patient age, sex, traditional risk factors, underlying disease, and concomitant medications were obtained from electronic medical records and laboratory data. Valvular heart diseases were defined according to angiographic, hemodynamic, or echocardiographic findings or the history of valvular surgery. Valvular regurgitation was defined as valvular disease when we identified greater than moderate regurgitation on color-flow Doppler echocardiography. Coronary artery disease was defined as positive stress test findings, coronary angiography, or coronary computed tomography angiography demonstrating stenosis of at least $75 \%$, a history of prior myocardial infarction, or a history of revascularization procedures. Nonischemic cardiomyopathies were defined as ventricular myocardial abnormalities in the absence of coronary artery disease or valvular, pericardial, or congenital heart disease. Tachycardia-induced cardiomyopathy was defined as the presence of no other cause of nonischemic cardiomyopathy, no LV hypertrophy, relatively normal LV dimensions, and the recovery of LV function after the control of tachycardia within 1-6 months [20]. The $\mathrm{CHADS}_{2}$ score (cardiac failure, hypertension, age $\geq 75$ years, diabetes, and previous stroke or transient ischemic attack) was used to measure stroke risk.

Echocardiography was performed on admission for all patients and was also performed in patients for whom cardiac function assessment was required during landiolol therapy. Echocardiographic data were obtained by an independent investigator (K.A.) who was blinded to the patients' data. The LV end-diastolic volume and LV end-systolic volume were measured in apical two- and four-chamber views. From these results, the LV ejection fraction (LVEF) was calculated using the biplane Simpson method. The cardiac chamber dimension on the M-mode echocardiogram was used to measure the LV end-diastolic dimension, LV endsystolic dimension, and left atrial dimension.

After hospital discharge, the patients were observed as outpatients at our hospital or their general practitioner's clinic at 1- to 3-month intervals. The patients were followed until death from any cause, loss to follow-up, or June 2018. Information regarding deceased patients was obtained from medical records, family members, the patients' general practitioners, and the hospitals to which the patients had been admitted. 


\subsection{Drug Dosing}

In our hospital, landiolol diluted with $5 \%$ glucose was administered continuously at an initial dosage of $1.0 \mu \mathrm{g} / \mathrm{kg} /$ min for $1 \mathrm{~min}$, followed by an infusion that was titrated from 1.0 to $10.0 \mu \mathrm{g} / \mathrm{kg} / \mathrm{min}$ until the target heart rate was less than 100 beats/min according to the recommendations of the ESC guidelines [13], and heart rate and blood pressure were monitored. The drug was then administered continuously at the maximum tolerable dose for each patient as a maintenance dose. Continuous electrocardiogram (ECG) monitoring was used during landiolol therapy. Blood pressure was measured frequently, particularly during the dose titration period. Blood pressure was measured automatically (BP8800SF, Omron Colin Co., Ltd., Tokyo, Japan) at approximately 6, 12 , and $24 \mathrm{~h}$ after the start of the continuous infusion of landiolol. If drug-related adverse events, such as excessive bradycardia and hypotension occurred, the landiolol dose was reduced or treatment was discontinued.

When landiolol treatment did not result in an adequate response in terms of the cardiac status, additional procedures such as cardioversion were performed at the physician's discretion.

\subsection{Endpoints}

The primary endpoints were changes in heart rate and systolic blood pressure from baseline during intravenous landiolol infusion. Secondary endpoints were the restoration of sinus rhythm and clinical outcome. We also assessed adverse events such as hypotension (defined as a systolic blood pressure of $<90 \mathrm{mmHg}$ ), bradycardia of $<40$ beats/min, ventricular tachyarrhythmias, or any other adverse events requiring the discontinuation of landiolol therapy.

\subsection{Statistical Analysis}

Data are presented as means and standard deviations or medians with ranges or frequencies. Student's t-test was used to assess differences between continuous variables. Categorical variables are reported as the observed number of patients (percentages) and were analyzed by either the chi-square test or Fisher's exact test, as appropriate. Heart rate and systolic blood pressure during landiolol therapy were compared to the baseline level of each parameter using two-way analysis of variance (ANOVA) for repeated measurements followed by the Bonferroni post hoc test. A two-sided $P$ value of $<0.05$ was considered to indicate significance throughout the analyses. All statistical analyses were performed with JMP Pro software, version 12.1.0 (SAS Institute, Cary, NC, USA).

\section{Results}

\subsection{Patient Characteristics}

The clinical characteristics of the patients are summarized in Table 1 . The mean age was $67 \pm 12$ years, and 36 patients were male. The mean heart rate was $141 \pm 17$ beats/min, and 40 patients were in New York Heart Association functional class III/IV. The mean LVEF was $41 \pm 13 \%$. Three patients received a concomitant administration of intravenous dobutamine and three patients received intra-aortic balloon pumping. The median maintenance dose of intravenous landiolol was $3.0(1.0-12.0) \mu \mathrm{g} / \mathrm{kg} / \mathrm{min}$, and the median treatment duration of intravenous landiolol was 5 (1-24) days. The median time from the start of landiolol therapy to attaining the median maintenance dose was $6(0-30) \mathrm{h}$.

\subsection{Heart Rate and Blood Pressure During Landiolol Treatment}

Figure 1 shows the changes in heart rate and systolic blood pressure from baseline in 67 patients during intravenous treatment with landiolol. Two-way repeated-measures ANOVA indicated that the heart rate significantly decreased during intravenous landiolol treatment compared to the baseline $(P<0.001)$. The heart rate significantly decreased after the start of intravenous landiolol treatment $(141 \pm 17$ beats/min at baseline to $99 \pm 20$ beats/min at $6 \mathrm{~h}, P<0.001$ ), and this effect persisted throughout intravenous landiolol treatment. The systolic blood pressure did not significantly decrease during intravenous landiolol treatment compared to the baseline as determined via two-way repeated-measures ANOVA. In particular, in four patients with severe systolic dysfunction (LVEF $\leq 30 \%$ ), intravenous landiolol treatment (maintenance dose range $1.0-10.0 \mu \mathrm{g} / \mathrm{kg} / \mathrm{min}$ ) did not decrease the blood pressure or LVEF (Table 2).

Among our patients, there were no significant differences in the maintenance dose of landiolol between the ADHF patients with LVEF $\geq 40 \%(n=41)$ and those with LVEF $<40 \%(n=26)$ (median maintenance dose: 3.0 (2.0-6.0) $\mu \mathrm{g} / \mathrm{kg} / \mathrm{min}$ vs. $4.0(2.8-8.0) \mu \mathrm{g} / \mathrm{kg} / \mathrm{min}$, respectively). However, there was a greater decrease in the achieved heart rate in the patients with LVEF $\geq 40 \%$ than those with LVEF $<40 \%(79 \pm 16$ beats/min vs. $88 \pm 14$ beats/ min, respectively, $P=0.017$ ).

\subsection{Outcome}

The clinical outcomes during and after intravenous landiolol infusion are shown in Fig. 2. Of the 67 patients who started intravenous landiolol, sinus rhythm was restored 
Table 1 Baseline characteristics in 67 patients with atrial tachyarrhythmias and acute decompensated heart failure

\begin{tabular}{|c|c|}
\hline Male $(n)$ & 36 \\
\hline Age (years) & $67 \pm 12$ \\
\hline \multicolumn{2}{|l|}{ Atrial tachyarrhythmias } \\
\hline Atrial fibrillation & 48 \\
\hline Atrial flutter & 7 \\
\hline Atrial tachycardia & 12 \\
\hline \multicolumn{2}{|l|}{ Blood pressure (mmHg) } \\
\hline Systolic & $116 \pm 19$ \\
\hline Diastolic & $68 \pm 16$ \\
\hline Heart rate (beats/min) & $141 \pm 17$ \\
\hline \multicolumn{2}{|l|}{ Underlying heart disease } \\
\hline Coronary artery disease & 15 \\
\hline Non-ischemic cardiomyopathies & 17 \\
\hline Valvular disease & 11 \\
\hline Tachycardia-induced cardiomyopathy & 8 \\
\hline Others & 16 \\
\hline \multicolumn{2}{|l|}{ NYHA functional class at admission } \\
\hline II/III/IV & $27 / 28 / 12$ \\
\hline $\operatorname{LVEF}(\%)$ & $41 \pm 13$ \\
\hline Pacemaker and/or ICD & 5 \\
\hline \multicolumn{2}{|l|}{$\mathrm{CHADS}_{2}$ score } \\
\hline $1 / 2 / 3 / 4 / \geq 5$ & $12 / 26 / 19 / 7 / 3$ \\
\hline \multicolumn{2}{|l|}{ Laboratory data at admission } \\
\hline White blood cell count $\left(\times 10^{3} / \mu \mathrm{l}\right)$ & $7.6 \pm 2.7$ \\
\hline Hemoglobin $(\mathrm{g} / \mathrm{dl})$ & $12.5 \pm 2.4$ \\
\hline Albumin $(\mathrm{g} / \mathrm{dl})$ & $3.6 \pm 0.8$ \\
\hline Total bilirubin (mg/dl) & $0.9 \pm 0.4$ \\
\hline BUN (mg/dl) & $27.2 \pm 13.7$ \\
\hline Creatinine (mg/dl) & $1.0(0.8-1.5)$ \\
\hline Sodium $(\mathrm{mEq} / \mathrm{l})$ & $136 \pm 16$ \\
\hline Potassium (mEq/l) & $4.4 \pm 0.6$ \\
\hline $\mathrm{CRP}(\mathrm{mg} / \mathrm{dl})$ & $0.7(0.2-3.6)$ \\
\hline Plasma BNP (pg/ml) & $468(277-896)$ \\
\hline \multicolumn{2}{|l|}{ Medications at admission } \\
\hline$\beta$-Blockers & 24 \\
\hline ACE inhibitors/ARBs & 28 \\
\hline MRAs & 11 \\
\hline Loop diuretics & 18 \\
\hline Amiodarone & 4 \\
\hline Other antiarrhythmic drugs & 4 \\
\hline Digoxin & 6 \\
\hline Warfarin & 14 \\
\hline Direct oral anticoagulants & 26 \\
\hline \multicolumn{2}{|l|}{ Treatment for ADHF } \\
\hline Intravenous loop diuretics & 31 \\
\hline Intravenous nitrates & 9 \\
\hline Intravenous carperitide & 6 \\
\hline Intravenous dobutamine & 3 \\
\hline Intravenous amiodarone & 1 \\
\hline Intravenous heparin & 24 \\
\hline
\end{tabular}

Table 1 (continued)

\begin{tabular}{ll} 
Noninvasive positive pressure ventilation & 7 \\
Intra-aortic balloon pumping & 3 \\
Percutaneous coronary intervention & 2 \\
\hline
\end{tabular}

Values are $\mathrm{n}$ or mean $\pm \mathrm{SD}$ or median (quartile)

$A C E$ angiotensin-converting enzyme, $A D H F$ acute decompensated heart failure, $A R B$ angiotensin II receptor blocker, $B N P$ brain natriuretic peptide, $B U N$ blood urea nitrogen, $C R P$ C-reactive protein, $I C D$ implantable cardioverter-defibrillator, $L V E F$ left ventricular ejection fraction, MRA mineralocorticoid receptor antagonist, NYHA New York Heart Association

spontaneously in $15(22 \%)$ patients and by electrical or pharmacological cardioversion in five patients during treatment with intravenous landiolol. Furthermore, sinus rhythm was restored in 22 patients who exhibited persistent ATAs using additional rhythm control treatments, such as oral amiodarone and catheter ablation after landiolol treatment. Eight patients experienced in-hospital death. Forty-one (69\%) of the 59 patients who were alive at discharge were in sinus rhythm. For the remaining 18 patients with persistent ATAs, $\beta$-blockers and digoxin were added as a rate control treatment (Table 3).

During the follow-up period of $16 \pm 12$ months, four patients died, and 12 patients experienced rehospitalization due to worsening HF after hospital discharge. Rehospitalization due to worsening HF occurred less frequently in patients who were discharged with sinus rhythm than in those discharged with ATAs (5/41 vs. $7 / 18, P=0.019)$ (Table 4). Of the 41 patients with sinus rhythm at discharge, 24 (59\%) patients experienced a recurrence of ATAs after discharge. Three of the 24 patients who experienced recurrent ATAs and two of the 17 patients without recurrent ATAs were rehospitalized due to worsening HF.

\subsection{Adverse Effects}

Landiolol treatment was discontinued in three patients because of the following adverse effects: hypotension in two patients (1.0 and $3.0 \mu \mathrm{g} / \mathrm{kg} / \mathrm{min})$ and liver dysfunction in one patient $(1.5 \mu \mathrm{g} / \mathrm{kg} / \mathrm{min})$.

\section{Discussion}

Our study in 67 patients with ATAs and ADHF who were treated with intravenous landiolol had the following findings: (1) intravenous landiolol reduced the heart rate without markedly reducing blood pressure or leading to a deterioration in HF or cardiac function, (2) sinus rhythm was spontaneously restored in 15 (22\%) patients during landiolol treatment, (3) patients with an LVEF of $\geq 40 \%$ showed a greater 
Fig. 1 Changes in heart rate (a) and systolic blood pressure (b) before and during intravenous landiolol infusion. $* P<0.001$

vs. baseline
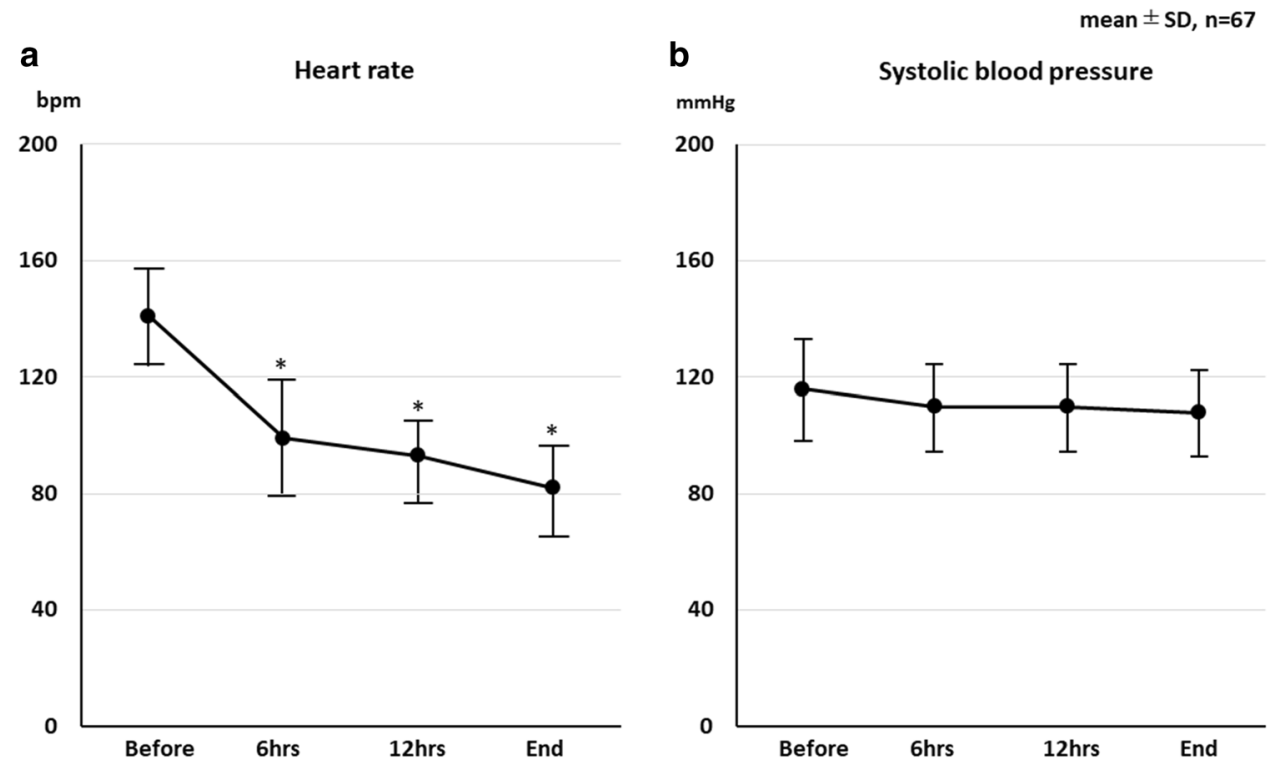

Table 2 Changes in heart rate, blood pressure, and echocardiographic parameters from baseline during intravenous landiolol infusion in four patients with LVEF $\leq 30 \%$

\begin{tabular}{|c|c|c|c|c|c|c|c|c|c|c|}
\hline \multirow{2}{*}{$\begin{array}{l}\text { Case } \\
\text { Age/sex }\end{array}$} & \multicolumn{2}{|c|}{ HR (beats/min) } & \multicolumn{2}{|c|}{$\mathrm{SBP}(\mathrm{mmHg})$} & \multicolumn{2}{|c|}{ LVDd (mm) } & \multicolumn{2}{|c|}{ LVEF (\%) } & \multicolumn{2}{|c|}{ LAD (mm) } \\
\hline & Baseline & Landiolol & Baseline & Landiolol & Baseline & Landiolol & Baseline & Landiolol & Baseline & Landiolol \\
\hline $53 / \mathrm{M}$ & 155 & 80 & 80 & 90 & 71 & 61 & 15 & 41 & 47 & 42 \\
\hline $67 / \mathrm{M}$ & 140 & 70 & 130 & 108 & 53 & 50 & 20 & 54 & 32 & 34 \\
\hline $80 / \mathrm{F}$ & 141 & 85 & 124 & 114 & 51 & 47 & 25 & 43 & 37 & 36 \\
\hline 68/M & 133 & 98 & 136 & 128 & 60 & 53 & 30 & 54 & 45 & 51 \\
\hline
\end{tabular}

$F$ female, $H R$ heart rate, $L A D$ left atrial dimension, $L V D d$ left ventricular end-diastolic dimension, $L V E F$ left ventricular ejection fraction, $M$ male, $S B P$ systolic blood pressure

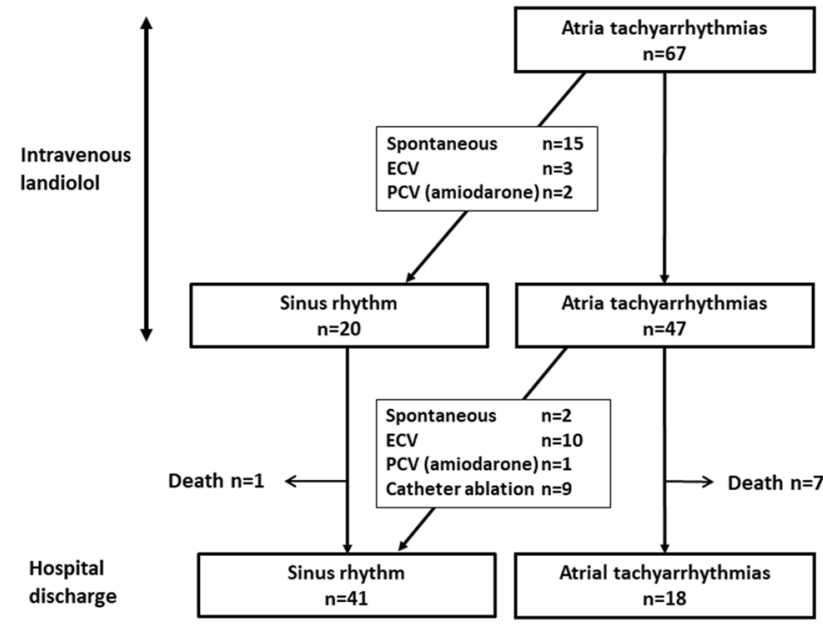

Fig. 2 Sinus rhythm restoration in patients during and after intravenous landiolol infusion. $E C V$ electrical cardioversion, $P C V$ pharmacological cardioversion
Table 3 Treatment in 59 patients who were alive at discharge

\begin{tabular}{lll}
\hline & $\begin{array}{l}\text { Sinus rhythm } \\
(n=41)\end{array}$ & $\begin{array}{l}\text { Atrial tachyarrhythmia } \\
(n=18)\end{array}$ \\
\hline Pacemaker and/or ICD & 8 & 2 \\
Medications & & \\
$\beta$-Blockers & 35 & 16 \\
ACE inhibitors/ARBs & 25 & 12 \\
MRAs & 15 & 8 \\
Loop diuretics & 24 & 15 \\
Amiodarone & 15 & 1 \\
Digoxin & 3 & 7 \\
Catheter ablation & 17 & 1 \\
\hline
\end{tabular}

\section{Values are $n$}

$A C E$ angiotensin-converting enzyme, $A R B$ angiotensin II receptor blocker, ICD implantable cardioverter-defibrillator, $M R A$ mineralocorticoid receptor antagonist 
Table 4 Summary of clinical outcomes

\begin{tabular}{lll}
\hline After landiolol treatment & Sinus rhythm $(n=20)$ & Atrial tachyarrhythmias $(n=47)$ \\
\hline In-hospital death & 1 & 7 \\
Cardiac cause & 1 & 3 \\
Non-cardiac cause & 0 & 4 \\
\hline After hospital discharge & Sinus rhythm $(n=41)$ & Atrial tachyarrhythmias $(n=18)$ \\
\hline Death & 3 & 1 \\
Cardiac cause & 1 & 0 \\
Non-cardiac cause & 2 & 1 \\
Rehospitalization due to worsening heart failure & 5 & 7 \\
\hline
\end{tabular}

Values are $n$

decrease in heart rate than those with an LVEF of $<40 \%$ despite a similar dose of landiolol, (4) approximately $70 \%$ of the discharged patients were in sinus rhythm, and (5) several landiolol-related adverse effects, such as hypotension, were observed.

\subsection{Rate Control in the Acute Setting}

In the acute setting of HF with rapid ATAs, $\beta$-blockers are useful for rate reduction and are preferred over digoxin due to their effectiveness in the setting of high sympathetic tone [9]. The use of $\beta$-blockers clearly requires an incremental dosage to achieve a heart rate that balances the need for rate control with other hemodynamic parameters [9]. B-Blockers have negative inotropic and blood pressure-lowering effects and are thus of limited use, especially when administered intravenously, in ADHF patients with reduced LV systolic function and/or low blood pressure.

In our study, the maintenance dose of landiolol was attained at a median of $6 \mathrm{~h}$ after the start of treatment and significantly reduced the heart rate without markedly reducing blood pressure. Landiolol has high cardioselectivity and exhibits a higher heart rate-lowering potency and a slightly more potent negative chronotropic effect with a less potent negative inotropic effect than esmolol [14]. The effect of landiolol appears early after the initiation of treatment and rapidly stabilizes because of its pharmacokinetic properties, such as its relatively small volume of distribution and short elimination time.

Kobayashi et al. reported that low doses (1.0-2.0 $\mu \mathrm{g} /$ $\mathrm{kg} / \mathrm{min}$ ) of intravenous landiolol reduced the heart rate while maintaining blood pressure in patients with rapid atrial fibrillation and ADHF without adverse events, such as hypotension or HF deterioration [21]. In addition, Adachi et al. reported that intravenous landiolol significantly reduced the heart rate during ATAs in patients with ADHF and a low LVEF despite the frequent concomitant use of intravenous inotropic drugs [22]. Of our patients, three received concomitant administration of intravenous dobutamine, and three received intra-aortic balloon pumping while their heart rates were reduced during landiolol therapy without leading to a marked reduction in blood pressure.

\subsection{LVEF $\geq \mathbf{4 0} \%$ Versus LVEF $<40 \%$}

The ESC guidelines state that the drug choice for acute heart rate control in AF depends on the LVEF; $\beta$-blockers are the first-line treatment for patients with an LVEF of $\geq 40 \%$ or $<40 \%$ [13]. Our patients with ATAs and ADHF included 24 patients with an LVEF of $\geq 40 \%$. In our study, there was a greater decrease in heart rate in patients with an LVEF of $\geq 40 \%$ than in those with an LVEF of $<40 \%$ despite a similar maintenance dose of landiolol. Kobayashi et al. reported that HF patients with an LVEF of $<50 \%$ experienced a greater decrease in the heart rate than HF patients with an LVEF of $\geq 50 \% 1-2 \mathrm{~h}$ after the start of landiolol treatment, although there was no difference at later time points [21]. Ozaki et al. reported that HF patients with an LVEF of $\geq 40 \%$ showed a greater decrease in heart rate than HF patients with an LVEF of $<40 \%$ after the start of a similar dose of landiolol [23]. Patients with an LVEF of $\geq 40 \%$ are more likely to experience the heart rate-lowering effect of landiolol than those with an LVEF of $<40 \%$. Particularly in patients with severe LV systolic dysfunction (LVEF $\leq 30 \%)$ and an inherent increased risk of adverse cardiac events and mortality, landiolol therapy could be continued without hemodynamic worsening, and the LVEF values of these patients were subsequently improved after landiolol therapy.

However, it may be important to characterize LV systolic function when initiating landiolol treatment [19]. Wada et al. reported that an extremely low LVEF $(<25 \%)$ was a predictor of inadequate heart-rate lowering during landiolol therapy and was associated with the development of adverse events, such as hypotension [24]. 


\subsection{Outcomes}

Landiolol has a role in reliably lowering the heart rate and improving hemodynamics and the condition of ADHF. As a result, $22 \%$ of the patients exhibited a spontaneously restored sinus rhythm, and when defibrillation was added, 42 of the 67 patients showed a restored sinus rhythm during hospitalization. In HF patients, sympathetic activity is increased, and the stimulation of $\beta$-adrenergic receptors alters the activity of several ion channels and transporters and leads to arrhythmogenesis related to cardiomyocyte $\mathrm{Ca}^{2+}$ handling $[25,26]$. B-Blockers operate through multiple antiarrhythmic mechanisms via changes in action potentials or membrane currents induced by the suppression of various ion channels. Landiolol may be effective in treating ATAs caused by an increased sympathetic drive in HF.

After landiolol therapy, further enhancements to the basic therapy for HF, such as the use of $\beta$-blockers and renin-angiotensin-system inhibitors, pharmacological or electrical cardioversion, and additional rhythm control strategies, such as oral amiodarone administration and catheter ablation, were provided. Forty-one (69\%) of the 59 patients who were alive at discharge were in sinus rhythm. Of these patients, 24 (59\%) patients experienced a recurrence of ATAs after discharge. However, since there was no difference in the incidence of rehospitalization due to worsening HF between patients with and without recurrent ATAs (3/24 vs. 2/17), recurrent AF might not be associated with a poorer prognosis of patients with recurrent ATAs. In patients with ATAs and ADHF, the restoration of sinus rhythm improves hemodynamics and cardiac function, and additional therapy for the restoration and preservation of sinus rhythm may lead to the prevention of HF exacerbation and the subsequent improvement in prognosis.

In the acute setting, there is a high recurrence in patients in whom initial cardioversion is successful [12]. In this situation, landiolol may be useful as the first-line therapy for improving the hemodynamic status by lowering the heart rate. After the cardiac status is stabilized, subsequent cardioversion and rhythm control therapy, such as catheter ablation, will be effective, although sinus rhythm cannot be restored during landiolol treatment. Of our patients who were alive at discharge, approximately $70 \%$ were in sinus rhythm; these patients showed a lower frequency of subsequent rehospitalization due to worsening HF. The restoration of sinus rhythm is essentially related to prognosis in patients after discharge. However, it is difficult to restore and maintain sinus rhythm in patients with deteriorated hemodynamics. It is necessary to stabilize hemodynamics by rapidly controlling the rate of ATAs in ADHF. Landiolol may reliably lower the heart rate during ATAs in ADHF patients without the deterioration of hemodynamics.
Patients with ATAs and ADHF often have a concomitant systemic illness, such as an infectious disease. Among our patients, more than half of the deaths that occurred were from non-cardiac causes. In these cases, the treatment of ATAs and HF does not necessarily improve the prognosis; however, it may contribute to stabilizing the circulation.

\subsection{Adverse Effects}

Wada et al. reported that hypotension was often observed (in approximately $10 \%$ of cases) as an adverse effect in patients with arrhythmia who received intravenous landiolol, and that hypotension was associated with a lower LVEF [24]. In our study, two patients experienced hypotension during therapy; however, their blood pressure recovered after the discontinuation of landiolol. During the use of landiolol, blood pressure should be carefully monitored in patients with low blood pressure or a low LVEF. Therefore, landiolol is useful as a rate control treatment for rapid ATAs in the acute setting of ADHF.

\subsection{Study Limitations}

There are several limitations in this study. First, this study was a single-center non-comparative, retrospective observational study. Therefore, treatment bias existed. We could not evaluate the relationship between the recurrence of ATAs and the outcome. It is difficult to obtain the exact time to the recurrence of ATAs or the frequency of ATAs based on the patient's symptoms and routine ECGs in clinical practice. Second, landiolol has been used only in Japan for 15 years, and the generalizability of our results to Japanese patients is limited to clinical practice in Europe and other countries. Third, the clinical characteristics of the subjects varied, and the number of subjects was small. Therefore, a subgroup analysis was not feasible.

\section{Conclusions}

Our results showed that intravenous landiolol reduced the heart rate without markedly decreasing blood pressure in patients with ATAs and ADHF. Furthermore, approximately $70 \%$ of the patients who were alive at discharge were in sinus rhythm, and these patients had a lower frequency of rehospitalization due to worsening HF during the mean 16-month follow-up.

\section{Compliance with Ethical Standards}

Conflict of interest T. Shiga received a speaker honorarium from ONO Pharmaceutical Co., Ltd. T. Shiga and A. Suzuki were investigators in 
the late phase II/phase III study ONO-1101-30. T. Shiga, A. Suzuki, and $\mathrm{K}$. Arai were investigators in the phase III study ONO-1162. Y. Matsui and N. Hagiwara have no conflicts of interest.

Funding This study did not receive funding from any agency in the public, commercial, or nonprofit sectors.

Open Access This article is distributed under the terms of the Creative Commons Attribution-NonCommercial 4.0 International License (http://creativecommons.org/licenses/by-nc/4.0/), which permits any noncommercial use, distribution, and reproduction in any medium, provided you give appropriate credit to the original author(s) and the source, provide a link to the Creative Commons license, and indicate if changes were made.

\section{References}

1. Nieuwlaat R, Eurlings LW, Cleland JG, Cobbe SM, Vardas PE, Capucci A, López-Sendòn JL, Meeder JG, Pinto YM, Crijns HJ. Atrial fibrillation and heart failure in cardiology practice: reciprocal impact and combined management from the perspective of atrial fibrillation: results of the Euro Heart Survey on atrial fibrillation. J Am Coll Cardiol. 2009;53:1690-8.

2. Li SJ, Sartipy U, Lund LH, Dahlström U, Adiels M, Petzold M, $\mathrm{Fu}$ M. Prognostic significance of resting heart rate and use of $\beta$-Blockers in atrial fibrillation and sinus rhythm in patients with heart failure and reduced ejection fraction: findings from the Swedish Heart Failure Registry. Circ Heart Fail. 2015;8:871-9.

3. Benjamin EJ, Wolf PA, D'Agostino RB, Silbershatz H, Kannel WB, Levy D. Impact of atrial fibrillation on the risk of death: the Framingham Heart Study. Circulation. 1998;98:946-52.

4. Wang TJ, Larson MG, Levy D, Vasan RS, Leip EP, Wolf PA, D'Agostino RB, Murabito JM, Kannel WB, Benjamin EJ. Temporal relations of atrial fibrillation and congestive heart failure and their joint influence on mortality: the Framingham Heart Study. Circulation. 2003;107:2920-5.

5. Crijns HJGM, Van Den Berg MP, Van Gelder IC, Van Veldhuisen DJ. Management of atrial fibrillation in the setting of heart failure. Eur Heart J. 1997;18:C45-9.

6. Van Den Berg MP, Tuinenburg AE, Crijns HJGM, Van Gelder IC, Gosselink AT, Lie KI. Heart failure and atrial fibrillation: current concepts and controversies. Heart. 1997;77:309-13.

7. Cha YM, Redfield MM, Shen WK, Gersh BJ. Atrial fibrillation and ventricular dysfunction: a vicious electromechanical cycle. Circulation. 2004;109:2839-43.

8. Mene-Afejuku TO, López PD, Akinlonu A, Dumancas C, Visco F, Mushiyev S, Pekler G. Atrial fibrillation in patients with heart failure: current state and future directions. Am J Cardiovasc Drugs. 2018;18:347-60.

9. Kotecha D, Piccini JP. Atrial fibrillation in heart failure: what should we do? Eur Heart J. 2015;36:3250-7.

10. Middlekauff HR, Stevenson WG, Stevenson LW. Prognostic significance of atrial fibrillation in advanced heart failure: a study of 390 patients. Circulation. 1991;84:40-80.

11. Crijns HJ, Tjeerdsma G, de Kam PJ, Boomsma F, van Gelder IC, van den Berg MP, van Veldhuisen DJ. Prognostic value of the presence and development of atrial fibrillation in patients with advanced chronic heart failure. Eur Heart J. 2000;21:1238-45.

12. Mayr A, Ritsch N, Knotzer H, Dünser M, Schobersberger W, Ulmer H, Mutz N, Hasibeder W. Effectiveness of direct-current cardioversion for treatment of supraventricular tachyarrhythmias, in particular atrial fibrillation, in surgical intensive care patients. Crit Care Med. 2003;31:401-5.

13. Ponikowski P, Voors AA, Anker SD, Bueno H, Cleland JGF, Coats AJS, Falk V, González-Juanatey JR, Harjola VP, Jankowska EA, Jessup M, Linde C, Nihoyannopoulos P, Parissis JT, Pieske B, Riley JP, Rosano GMC, Ruilope LM, Ruschitzka F, Rutten FH, van der Meer P, ESC Scientific Document Group. 2016 ESC guidelines for the diagnosis and treatment of acute and chronic heart failure: the Task Force for the diagnosis and treatment of acute and chronic heart failure of the European Society of Cardiology (ESC) developed with the special contribution of the Heart Failure Association (HFA) of the ESC. Eur Heart J. 2016;37:2129-200.

14. Syed YY. Landiolol: a review in tachyarrthythmias. Drugs. 2018;78:377-88.

15. Domanovits H, Wolzt M, Stix G. Landiolol: pharmacology and its use for rate control in atrial fibrillation in an emergency setting. Eur Heart J Suppl. 2018;20(Suppl A):A1-3.

16. Plosker GL. Landiolol: a review of its use in intraoperative and postoperative tachyarrhythmias. Drugs. 2013;73:959-77.

17. Iguchi S, Iwamura H, Nishizaki M, Hayashi A, Senokuchi K, Kobayashi K, Sakaki K, Hachiya K, Ichioka Y, Kawamura M. Development of a highly cardioselective ultra short-acting betablocker, ONO-1101. Chem Pharm Bull (Tokyo). 1992;40:1462-9.

18. Sasao J, Tarver SD, Kindscher JD, Taneyama C, Benson KT, Goto H. In rabbits, landiolol, a new ultra-short-acting beta-blocker, exerts a more potent negative chronotropic effect and less effect on blood pressure than esmolol. Can J Anaesth. 2001;48:985-9.

19. von Haehling S, Bělohlávek J, Er F, Gassanov N, Guarracino F, Bouvet O. Landiolol for rate control management of atrial fibrillation in patients with cardiac dysfunction. Eur Heart J Suppl. 2018;20(Suppl A):A19-24.

20. Gupta S, Figueredo VM. Tachycardia mediated cardiomyopathy: pathophysiology, mechanisms, clinical features and management. Int J Cardiol. 2014;172:40-6.

21. Kobayashi S, Murakami W, Myoren T, Tateishi H, Okuda S, Doi M, Nao T, Wada Y, Matsuzaki M, Yano M. A low-dose $\beta 1$-blocker effectively and safely slows the heart rate in patients with acute decompensated heart failure and rapid atrial fibrillation. Cardiology. 2014;127:105-13.

22. Adachi T, Sato A, Baba M, Hiraya D, Hasegawa T, Kuroki K, Hoshi T, Aonuma K. Novel use of the ultra-short-acting intravenous $\beta 1$-selective blocker landiolol for supraventricular tachyarrhythmias in patients with congestive heart failure. Heart Vessels. 2014;29:464-9.

23. Ozaki T, Yamada T, Morita T, Furukawa Y, Tamaki S, Iwasaki Y, Kawasaki M, Kikuchi A, Kondou T, Sato Y. Urgent control of rapid atrial fibrillation using landiolol in patients with acute decompensated heart failure with reduced or preserved left ventricular ejection fraction. Eur Heart J. 2016;37(Abstract Supplement): 1319.

24. Wada Y, Aiba T, Tsujita Y, Itoh H, Wada M, Nakajima I, Ishibashi K, Okamura H, Miyamoto K, Noda T, Sugano Y, Kanzaki H, Anzai T, Kusano K, Yasuda S, Horie M, Ogawa H. Practical applicability of landiolol, an ultra-short-acting $\beta 1$-selective blocker, for rapid atrial and ventricular tachyarrhythmias with left ventricular dysfunction. J Arrhythm. 2016;32:82-8.

25. Rubart M, Zipes D. Mechanisms of sudden cardiac death. J Clin Investig. 2005;115:2305-15.

26. Chen PS, Chen LS, Fishbein MC, Lin SF, Nattel S. Role of the autonomic nervous system in atrial fibrillation: pathophysiology and therapy. Circ Res. 2014;114:1500-15. 